\title{
Editorials
}

\section{Cardiac surgery and intrathecal/epidural techniques: at the crossroads?}

Mark A. Chaney MD

$\mathrm{U}$ TILIZATION of intrathecal and epidural anesthesia and analgesia techniques in patients undergoing cardiac surgery has increased. $^{1,2}$ Potential benefits include improved postoperative analgesia, attenuation of the stress response to surgery, and induction of thoracic cardiac sympathectomy. These potential clinical benefits may reduce perioperative morbidity and mortality. However, substantial controversy persists regarding the risk:benefit ratio of intrathecal and epidural instrumentation in cardiac surgical patients who subsequently receive perioperative anticoagulation.

In this issue of the Journal, Jacobsohn and associates $^{3}$ present the findings of their small $(n=43)$, prospective, randomized, blinded, placebo-controlled clinical study investigating the potential clinical benefits of intrathecal morphine in healthy patients undergoing elective cardiac surgery with cardiopulmonary bypass. The intrathecal injection (morphine or placebo) was administered immediately prior to induction of general anesthesia and perioperative care was appropriately standardized. Extubation times were equivalent between the two groups (approximately 40 min). Patients receiving intrathecal morphine exhibited enhanced postoperative analgesia, as evidenced by decreased morphine requirements and decreased visual analogue pain scores. Both groups exhibited substantial pulmonary dysfunction (per spirometry) during the immediate postoperative period yet dysfunction was less pronounced in the patients receiving intrathecal morphine. Postoperative arterial blood gases and atelectasis scores were equivalent between the two groups, as were analgesic satisfaction, hospital satisfaction, and mean hospital length of stay. Thus, intrathecal morphine provided only enhanced post- operative analgesia (yet no enhancement of analgesic satisfaction). Such findings are not new (similar results have been previously published) yet present an opportunity to critically reassess the current use of intrathecal and epidural techniques in patients undergoing cardiac surgery.

Use of intrathecal and epidural techniques in patients undergoing cardiac surgery certainly enhances postoperative analgesia, may attenuate the stress response to surgery, and may induce thoracic cardiac sympathectomy. Because of enhanced flexibility (and more potential clinical benefits), most recent clinical investigations have focused on the use of thoracic epidural techniques. Intrathecal morphine was initially used in patients undergoing cardiac surgery in 1980. Since then, numerous clinical investigations (some prospective, randomized, blinded, placebo-controlled) attest to this technique's ability to induce reliable postoperative analgesia. However, neither intrathecal opioids or local anesthetics reliably attenuate the stress response associated with cardiac surgery or reliably induce perioperative thoracic cardiac sympathectomy. In contrast to intrathecal techniques, the presence of a catheter in the epidural space allows an almost infinite number of possibilities regarding potential drugs and dosing schedules to obtain specific goals. The initial description of thoracic epidural anesthesia and analgesia applied to a cardiac surgical patient occurred in 1954. Numerous clinical investigations (none well-controlled) attest to the ability of thoracic epidural opioids and local anesthetics to reliably induce postoperative analgesia in patients after cardiac surgery. Administration of thoracic epidural local anesthetics (not opioids) has the potential to attenuate the stress response associated with the perioperative period and

From the Department of Anesthesia and Critical Care, University of Chicago, Chicago, Illinois, USA.

Address correspondence to: Dr. Mark A. Chaney, Department of Anesthesia and Critical Care, University of Chicago, 5841 South

Maryland Avenue, MC 4028, Chicago, IL 60637, USA. Phone: 773-702-5951; Fax: 773-834-0063; E-mail: mchaney@dacc.uchicago.edu 
induce thoracic cardiac sympathectomy. While still controversial, suggested clinical benefits of thoracic epidural techniques in cardiac surgical patients include enhanced postoperative analgesia, improved postoperative pulmonary function, facilitation of early extubation, improved myocardial protection, decreased postoperative arrhythmias, improved postoperative renal function, and decreased postoperative confusion. ${ }^{4-7}$

Whether or not intrathecal and epidural techniques truly affect morbidity and mortality in patients undergoing cardiac surgery remains to be determined. Essentially all reports of intrathecal and epidural techniques in patients undergoing cardiac surgery involve small numbers of patients and few of these studies (if any) are well-designed. Prospective, randomized, blinded, placebo-controlled clinical investigations exist examining intrathecal techniques, yet no such clinical investigations exist examining epidural techniques. When critically reviewed, this body of literature suggests that these techniques reliably induce enhanced postoperative analgesia yet (at the current time) have no clinically important effect on morbidity and mortality. ${ }^{1,2}$

A recently-published meta-analysis provides some perspective. ${ }^{8}$ These authors, via Medline and other databases, searched for randomized controlled trials in patients undergoing coronary artery bypass surgery with cardiopulmonary bypass. Fifteen trials enrolling 1,178 patients were included for thoracic epidural analysis and 17 trials enrolling 668 patients were included for intrathecal analysis. Thoracic epidural techniques did not affect the incidence of mortality or myocardial infarction, yet appeared to reduce risk for dysrhythmias, reduce risk for pulmonary complications, reduce time to tracheal extubation, and reduce analogue pain scores. Intrathecal techniques did not affect incidence of mortality, myocardial infarction, dysrhythmias, or time to tracheal extubation, and only modestly decreased systemic morphine use and pain scores, while increasing incidence of pruritus. These authors concluded that central neuraxial analgesia does not affect mortality or myocardial infarction after coronary artery bypass grafting yet is potentially associated with earlier tracheal extubation, decreased pulmonary complications and cardiac dysrhythmias, and reduced pain scores. However, the authors also note that the majority of potential clinical benefits offered by central neuraxial analgesia (earlier extubation, decreased dysrhythmias, enhanced analgesia) may be achieved by other methods (fast-track anesthesia techniques, betablockers, amiodarone, other analgesic drugs) that do not entail risks associated with regional anesthesia.
Which leads us to risk. The most troubling and undesirable drug effect of intrathecal and epidural local anesthetics is hypotension. Epidural anesthesia and analgesia may either mask myocardial ischemia or initiate myocardial ischemia (through alterations in sympathetic-parasympathetic balance). The four clinically relevant undesirable drug effects of intrathecal and epidural opioids (pruritus, nausea and vomiting, urinary retention, respiratory depression) are well known. ${ }^{9}$ The optimal dose of intrathecal or epidural opioid in this setting, along with the optimal intraoperative baseline anesthetic, that will provide significant postoperative analgesia yet not delay tracheal extubation in the immediate postoperative period, is difficult to predict. In contrast to intrathecal and epidural opioids, epidural local anesthetics (which initiate no respiratory depression) should not delay tracheal extubation in the immediate postoperative period. Regarding hematoma formation, most investigators agree that risk is increased when intrathecal or epidural instrumentation is performed in patients prior to systemic heparinization required for cardiac surgery (absolute degree is controversial). Additionally, many cardiac surgical patients are administered drugs that affect coagulation during the preoperative period and/or require anticoagulation during the postoperative period. A recently published extensive mathematical analysis estimated the maximum risk of hematoma may be as high as $1: 2,400$ for intrathecal injection and 1:1,000 for epidural instrumentation in this setting (99\% confidence levels). ${ }^{10}$ In 2004, the first ever case report of an epidural hematoma with a thoracic epidural catheter inserted in a patient prior to cardiac surgery was published. ${ }^{11}$ Furthermore, this author is aware of at least three additional cases of catastrophic (permanent paralysis) epidural hematoma formation in patients who had thoracic epidural catheters inserted for elective cardiac surgery within the past five years in the United States alone (none published). Additionally, thromboembolic complications (stroke) may occur during the postoperative period when normalization of coagulation parameters (in a patient requiring anticoagulation) are achieved to safely remove the epidural catheter. ${ }^{12}$ Thus, bleeding and/or thromboembolic complications associated with these techniques in this setting are real and potentially catastrophic.

Multiple factors interact during the perioperative period that affect outcome and quality of life following cardiac surgery (Table). The list of factors in the Table are presented in no particular order. Obviously, depending on specific clinical situations, certain factors will be more important than others. It 
TABLE Factors affecting outcome following cardiac surgery

Type and quality of surgical intervention

Extent of postoperative neurologic dysfunction

Extent of postoperative myocardial dysfunction

Extent of postoperative pulmonary dysfunction

Extent of postoperative renal dysfunction

Extent of postoperative coagulation abnormalities

Quality of postoperative analgesia

Extent of systemic inflammatory response

is extremely difficult (if not impossible) to determine exactly how important attaining adequate postoperative analgesia truly is in relation to all of these clinical factors surrounding a patient undergoing cardiac surgery. Furthermore, a clear link between "adequate" or "high-quality" postoperative analgesia and outcome in patients following cardiac surgery has yet to be established. ${ }^{13-16}$ However, despite the absence of substantiating scientific evidence, most clinicians intuitively believe that attaining high-quality postoperative analgesia is important because it may prevent adverse hemodynamic, metabolic, immunologic, and hemostatic alterations. While many analgesic techniques are available, iv systemic opioids form the cornerstone of post-cardiac surgery analgesia (used for many years with excellent results). Lastly, the American Society of Anesthesiologists Task Force on Acute Pain Management in the Perioperative Setting offers sound advice. ${ }^{17}$ It recommends that anesthesiologists who manage perioperative pain should utilize analgesic therapeutic options only after thoughtfully considering the risks and benefits for the individual patient. The therapy (or therapies) selected should reflect the individual anesthesiologist's expertise, as well as the capacity for safe application of the chosen modality in each practice setting. This capacity includes the ability to recognize and treat adverse effects that emerge after initiation of therapy.

The only clear benefit of utilizing intrathecal or epidural techniques in patients undergoing cardiac surgery is enhanced postoperative analgesia. However, such enhanced analgesia (by any means) does not ensure improved outcome. The risks associated with these techniques in this setting are real, potentially catastrophic, and are beginning to emerge. After contemplating all of the factors that affect outcome following cardiac surgery (Table) and thoughtfully assessing the risk:benefit ratio, the question must be asked: is it time to abandon intrathecal and epidural techniques in patients undergoing cardiac surgery?

\section{Cardiochirurgie et tech- niques intrathécale/ péridurale : sommes- nous à la croisée des chemins?}

Il y a une augmentation de l'utilisation de techniques d'anesthésie et d'analgésie intrathécale et péridurale chez des patients de cardiochirurgie. ${ }^{1,2}$ Il y a, parmi les avantages potentiels, l'amélioration de l'analgésie postopératoire, l'atténuation de la réaction de stress à la chirurgie et l'induction de sympathectomie cardiaque thoracique. Ces avantages peuvent réduire la morbidité et la mortalité périopératoires. Mais une importante controverse persiste en ce qui concerne le rapport risque/bénéfice de l'instrumentation intrathécale et péridurale chez les patients de cardiochirurgie qui reçoivent subséquemment une anticoagulation périopératoire.

Dans le numéro actuel du Journal, Jacobsohn et ses collaborateurs ${ }^{3}$ présentent les résultats de leur étude clinique prospective, randomisée, à l'insu et contrôlée contre placebo, comportant un faible échantillon $(n=43)$ et réalisée pour explorer les bénéfices cliniques potentiels de l'administration intrathécale de morphine chez des patients sains qui doivent subir une intervention cardiaque avec circulation extracorporelle. L'injection intrathécale de morphine ou d'un placebo a été faite immédiatement avant l'induction de l'anesthésie générale et la prise en charge périopératoire a été normalisée de manière appropriée. L'extubation a eu lieu 40 min environ après l'opération chez les patients des deux groupes. Les patients sous morphine intrathécale ont bénéficié d'une meilleure analgésie postopératoire comme le prouvent les demandes moins importantes de morphine et les plus bas scores de douleur à l'échelle visuelle analogique. Immédiatement après l'opération, on a relevé dans les deux groupes, par spirométrie, une dysfonction pulmonaire substantielle, moins prononcée toutefois chez les patients recevant la morphine intrathécale. La gazométrie postopératoire du sang artériel et les scores d'atélectasie ont été équivalents dans les deux groupes, de même que la satisfaction reliée à l'analgésie et aux services hospitaliers et la longueur moyenne du séjour à l'hôpital. La morphine intrathécale n'a donc procuré qu'une amélioration de 
l'analgésie postopératoire, non pas de la satisfaction de l'analgésie. Ces résultats ne sont pas nouveaux, mais ils permettent de réévaluer de façon critique l'usage courant des techniques intrathécales et péridurales en cardiochirurgie.

L'utilisation de techniques intrathécales et péridurales en cardiochirurgie améliore certainement l'analgésie postopératoire, peut atténuer la réaction de stress à la chirurgie et induire une sympathectomie cardiaque thoracique. Étant donné l'amélioration de la flexibilité, et d'autres bénéfices cliniques potentiels, les recherches les plus récentes ont surtout porté sur les techniques péridurales thoraciques. C'est en 1980 qu'a débuté l'usage de morphine intrathécale en chirurgie cardiaque. Depuis, de nombreux essais cliniques, dont certains étaient prospectifs, randomisés, à l'insu et contrôlés contre placebo, montrent que cette technique peut induire une analgésie postopératoire efficace. Cependant, ni les opioïdes intrathécaux, ni les anesthésiques locaux ne diminuent de façon fiable la réaction de stress associée à la cardiochirurgie ou n'induisent régulièrement de sympathectomie cardiaque thoracique périopératoire. Par contre, la présence de cathéter dans l'espace péridural permet d'utiliser un nombre presque infini de médicaments et de programmes de dosages potentiels selon des objectifs spécifiques. L'anesthésie et l'analgésie péridurales thoraciques appliquées à la cardiochirurgie date de 1954. Puis, de nombreuses recherches cliniques, non bien contrôlées, montrent la capacité des opiö̈des périduraux thoraciques et des anesthésiques locaux à induire efficacement l'analgésie postopératoire chez des patients de cardiochirurgie. L'administration péridurale d'anesthésiques locaux, non d'opioïdes, peut atténuer la réaction de stress périopératoire et induire la sympathectomie cardiaque thoracique. Les avantages cliniques, encore controversés, des techniques péridurales thoraciques en cardiochirurgie comprennent l'amélioration de l'analgésie et de la fonction pulmonaire postopératoires, la facilitation de l'extubation précoce, l'amélioration de la protection myocardique, la diminution des arythmies postopératoires, l'amélioration de la fonction rénale postopératoire et la diminution de la confusion postopératoire. ${ }^{4-7}$

Il reste encore à découvrir si les techniques intrathécales et péridurales ont un véritable effet sur la morbidité et la mortalité des patients de cardiochirurgie. Tous les articles sur l'usage de techniques intrathécales et péridurales en cardiochirurgie ne comptent qu'un petit nombre de sujets et peu d'études, s'il en est, sont bien conçues. Des essais prospectifs, randomisés, à l'insu et contrôlés contre placebo ont été réalisés pour explorer les techniques intrathécales, mais aucun sur les techniques péridurales. Une revue critique de l'ensemble de ces recherches suggère que ces techniques induisent de façon fiable une meilleure analgésie postopératoire, même si pour le moment elles ne modifient pas beaucoup la morbidité et la mortalité. ${ }^{1,2}$

Une méta-analyse, publiée récemment, fournit une certaine perspective. ${ }^{8}$ Les auteurs ont utilisé, entre autres, la base Medline pour chercher des essais randomisés et contrôlés auprès de patients devant subir un pontage aortocoronarien avec circulation extracorporelle. Quinze essais comprenant 1178 patients ont été inclus pour analyse de l'anesthésie péridurale thoracique et 17 essais auprès de 668 patients pour analyse de l'anesthésie intrathécale. Les techniques péridurales thoraciques ne modifient pas l'incidence de mortalité ou d'infarctus du myocarde, mais semblent réduire le risque de dysrythmies et de complications pulmonaires, hâter l'extubation endotrachéale et diminuer les scores de douleur à l'échelle analogique. Les techniques intrathécales n'ont pas modifié l'incidence de mortalité, d'infarctus myocardique, de dysrythmies, ni hâter l'extubation. Elles n'ont que modestement fait baisser l'usage de morphine systémique et les scores de douleur, tandis qu'elles ont accru la fréquence de prurit. Les auteurs ont conclu que l'analgésie neuraxiale centrale ne modifie pas la mortalité ou l'infarctus du myocarde après le pontage aortocoronarien, mais qu'elle peut être liée à l'extubation endotrachéale précoce, à moins de complications pulmonaires et de dysrythmies cardiaques, et à des scores de douleur réduits. Toutefois, les auteurs ont aussi noté que la majorité des avantages cliniques possibles de l'analgésie neuraxiale centrale pouvaient être obtenus avec d'autres méthodes, comme les techniques d'anesthésie en régime accéléré, les bêta-bloquants, l'amiodarone et d'autres analgésiques qui ne comportent pas les risques associés à l'anesthésie régionale.

Ce qui nous amène à parler de risque. L'effet le plus inquiétant et le plus indésirable des anesthésiques locaux intrathécaux et périduraux est l'hypotension. L'anesthésie et l'analgésie péridurales peuvent masquer l'ischémie myocardique ou l'instaurer, par des altérations de l'équilibre sympathique-parasympathique. Les quatre effets indésirables cliniquement significatifs des opioïdes intrathécaux et périduraux sont bien connus : le prurit, les vomissements, la rétention urinaire et la dépression respiratoire. ${ }^{9}$ Dans ce contexte, il est difficile de prévoir quelle sera la dose optimale d'opioïde intrathécal ou péridural et l'anesthésique de base peropératoire optimal pouvant fournir une analgésie postopératoire significative sans retarder l'extubation endotrachéale postopératoire immédiate. 
Par opposition aux opioïdes intrathécaux et périduraux, les anesthésiques locaux, qui ne provoquent pas de dépression respiratoire, ne devraient pas retarder l'extubation endotrachéale postopératoire immédiate. Concernant la formation d'hématome, la plupart des chercheurs croient que le risque est accru quand une instrumentation intrathécale ou péridurale est utilisée avant l'héparinisation systémique nécessaire à la cardiochirurgie (l'héparinisation de degré absolu est controversée). De plus, de nombreux patients de cardiochirurgie reçoivent des médicaments qui affectent la coagulation pendant la période préopératoire et/ou exigent une anticoagulation postopératoire. Une vaste analyse mathématique récemment publiée a estimé le risque maximal d'hématome dans cette situation à 1:2 400 injections intrathécales et à 1:1 000 anesthésies péridurales (niveaux de confiance de $99 \%$ ). ${ }^{10}$ En 2004, un premier article fait état d'un cas d'hématome péridural en présence d'un cathéter péridural thoracique inséré avant la chirurgie cardiaque. ${ }^{11}$ L'auteur est au courant aussi d'au moins trois autres cas non publiés d'hématome péridural catastrophique, provoquant une paralysie permanente, chez des patients qui ont eu un cathéter péridural thoracique pour une intervention cardiaque réglée au cours des cinq dernières années aux États-Unis. Des complications thromboemboliques postopératoires peuvent survenir après la normalisation des paramètres de coagulation chez un patient qui reçoit une coagulothérapie pour permettre le retrait sécuritaire du cathéter péridural. ${ }^{12}$ Les saignements et/ou les complications thromboemboliques liés à ces techniques sont alors bien réels et peuvent être catastrophiques.

De multiple facteurs interagissent pendant la période périopératoire et affectent l'évolution du patient et sa qualité de vie à la suite d'une intervention cardiaque (Tableau). La liste y est présentée sans un ordre particulier. Évidemment, selon les situations cliniques spécifiques, certains facteurs seront plus importants que d'autres. Il est très difficile, sinon impossible, de déterminer exactement jusqu'où l'atteinte d'une analgésie postopératoire adéquate est vraiment en relation avec tous ces facteurs cliniques autour de la cardiochirurgie. De plus, un lien manifeste n'a pas encore été établi entre l'analgésie postopératoire «adéquate» ou de «haute qualité» et l'évolution des patients après la chirurgie cardiaque. ${ }^{13-16}$ Cependant, malgré l'absence de preuve scientifique établie, la majorité des cliniciens croient intuitivement qu'il est important d'obtenir une analgésie postopératoire de haute qualité parce que cela peut empêcher les changements hémodynamiques, métaboliques, immunologiques et hémostatiques indésirables. Même si de
TABLEAU Facteurs qui influencent les suites opératoires en cardiochirurgie

Type et qualité de l'intervention chirurgicale

Étendue de la dysfonction neurologique postopératoire

Étendue de la dysfonction myocardique postopératoire

Étendue de la dysfonction pulmonaire postopératoire

Étendue de la dysfonction rénale postopératoire

Étendue des anomalies de coagulation postopératoires

Qualité de l'analgésie postopératoire

Étendue de la réaction inflammatoire systémique

nombreuses techniques analgésiques sont possibles, les opioïdes systémiques iv forment la pierre angulaire de l'analgésie postopératoire en cardiochirurgie. Utilisés depuis de nombreuses années, ils présentent d'excellents résultats. Finalement, l'American Society of Anesthesiologists Task Force on Acute Pain Management in the Perioperative Setting recommande judicieusement ${ }^{17}$ que les anesthésiologistes qui traitent la douleur périopératoire utilisent l'analgésie seulement après avoir fait un examen minutieux des risques et bénéfices pour chaque patient. Qu'il s'agisse d'un ou de plusieurs traitements, il doit correspondre à l'expérience individuelle de l'anesthésiologiste autant qu'à sa capacité d'appliquer la modalité choisie dans chaque circonstance. Cette capacité inclut l'habileté à reconnaître et à traiter les effets indésirables survenant après le début du traitement.

Le seul avantage évident des techniques intrathécale ou péridurale chez des patients de cardiochirurgie est l'amélioration de l'analgésie postopératoire. Cependant, cette analgésie améliorée, par quelque moyen que ce soit, ne donne pas l'assurance d'une meilleure évolution. Les risques associés à ces techniques y sont réels, potentiellement catastrophiques, et commencent à émerger. Après avoir examiné tous les facteurs qui agissent sur les résultats de la chirurgie cardiaque (Tableau) et avoir évaluer avec attention le rapport risque/bénéfice, il y a une question à poser : est-il temps d'abandonner les techniques intrathécale et péridurale chez les patients qui subissent une intervention cardiaque?

\section{References}

1 Chaney MA. Regional Anesthesia for Cardiothoracic Surgery. Baltimore, MD: Lippincott Williams \& Wilkins; 2002: 1-167.

2 Chaney $M A$. Intrathecal and epidural anesthesia and analgesia for cardiac surgery. Anesth Analg 1997; 84: 1211-21. 
3 Jacobsobn E, Lee TW, Amadeo RJ, et al. The University of Manitoba Health Sciences Centre for Cardia Anesthesia Group. Low-dose intrathecal morphine does not delay early extubation after cardiac surgery. Can J Anesth 2005; 52: 848-57.

4 Barrington MJ, Kluger R, Watson R, Scott DA, Harris $K J$. Epidural anesthesia for coronary artery bypass surgery compared with general anesthesia alone does not reduce biochemical markers of myocardial damage. Anesth Analg 2005; 100: 921-8.

5 Royse C, Royse A, Soeding, Blake D, Pang J. Prospective randomized trial of high thoracic epidural analgesia for coronary artery bypass surgery. Ann Thorac Surg 2003; 75: 93-100.

6 Priestley MC, Cope L, Halliwell R, et al. Thoracic epidural anesthesia for cardiac surgery: the effects on tracheal intubation time and length of hospital stay. Anesth Analg 2002; 94: 275-82.

7 Scott NB, Turfrey DJ, Ray DA, et al. A prospective randomized study of the potential benefits of thoracic epidural anesthesia and analgesia in patients undergoing coronary artery bypass grafting. Anesth Analg 2001; 93: 528-35.

8 Lin SS, Block BM, Wu CL. Effects of perioperative central neuraxial analgesia on outcome after coronary artery bypass surgery. A meta-analysis. Anesthesiology 2004; 101: 153-61.

9 Chaney MA. Side effects of intrathecal and epidural opioids. Can J Anaesth 1995; 42: 891-903.

10 Ho AM, Chung DC, Joynt GM. Neuraxial blockade and hematoma in cardiac surgery. Estimating the risk of a rare adverse event that has not (yet) occurred. Chest 2000; 117: 551-5.

11 Rosen DA, Hawkinberry DW, Rosen KR, Gustafson RA, Hogg JP, Broadman LM. An epidural hematoma in an adolescent patient after cardiac surgery. Anesth Analg 2004; 98: 966-9.

12 Chaney MA, Labovsky JK. Case report of thoracic epidural anesthesia and cardiac surgery: balancing postoperative risks associated with hematoma formation and thromboembolic phenomenon. J Cardiothorac Vasc Anesth 2005 (in press).

13 Myles PS, Hunt JO, Fletcher H, Solly R, Woodward D, Kelly $S$. Relation between quality of recovery in hospital and quality of life at 3 months after cardiac surgery. Anesthesiology 2001; 95: 862-7.

14 Fleron MH, Weiskopf RB, Bertrand M, et al. A comparison of intrathecal opioid and intravenous analgesia for the incidence of cardiovascular, respiratory, and renal complications after abdominal aortic surgery. Anesth Analg 2003; 97: 2-12.

$15 W u C L$, Raja $S N$. Optimizing postoperative analgesia. The use of global outcome measures (Editorial).
Anesthesiology 2002; 97: 533-4.

16 Beattie WS, Badner NH, Choi P. Epidural analgesia reduces postoperative myocardial infarction: a metaanalysis. Anesth Analg 2001; 93: 853-8.

17 American Society of Anesthesiologists Task Force on Acute Pain Management. Practice guidelines for acute pain management in the perioperative setting. An updated report by the American Society of Anesthesiologists Task Force on Acute Pain Management. Anesthesiology 2004; 100: 1573-81. 